\title{
UNIFICACIÓN Y COHESIÓN DEL CONTINENTE EUROPEO. PRINCIPALES INSTRUMENTOS JURÍDICOS Y ECONÓMICOS PARA LA AMPLIACIÓN AL ESTE
}

\section{Elena F. PÉREZ CARRILLO*}

RESUMEN: Durante los primeros años del siglo XXI, la Unión Europea conocerá un proceso de ampliación sin precedentes. De la actual Europa de los 15, pasará a contar con 27, 28 o más miembros. Este reto supone tener en cuenta la entrada de países con variadas culturas y con sistemas socio-estructurales muy diferentes a los de Europa Occidental. Los mecanismos jurídicos, políticos y financieros para la preparación a la adhesión están en marcha. La adaptación al acervo comunitario, la cohesión económica y social, se encuentra entre los elementos que necesitan mayor atención y seguimiento para lograr el éxito.

ABSTRACT: During the early years of the XXIst Century the European Union will experience an unprecedented enlargement process. It will be transformed from today's Europe-15 into an organisation with 27, 28, or more Member States. This challenge will involve taking into account many new different cultural and socio economic backgrounds which are diverse from the current characteristics of Western Europe's societies. The article analyses the legal, political and financial instruments designed to facilitate the enlargement process. The author argues that cultural adaptation and social and economic cohesion have to be closely monitored in order to achieve a successful enlargement process.

RÉSUMÉ: Pendant les premières années du XXI siècle, l'Union Européenne connaîtra un processus de croissance sans précédent. De l'actuelle Europe des quinze elle passera à 27 ou 28 membres. L'entrée des nouveaux pays constitue un défi et cela suppose la prise en compte des diverses cultures et systèmes sociaux structurels très différents de ceux de l'Europe occidentale. Les mécanismes juridiques, politiques et financiers de préparation à l'adhésion sont en marche. L'adaptation au patrimoine communautaire, la cohésion économique et sociale se trouvent parmi les éléments les plus importants pour réussir l'intégration.

* Universidad Santiago de Compostela. 
SUMARIO: I. Integración europea e integraciones durante el siglo XX. II. Cohesión en una Europa ampliada. III. Financiación de la preadhesión. IV. Conclusiones. V. Bibliografía.

\section{INTEGRACIÓN EUROPEA E INTEGRACIONES DURANTE EL SIGLO XX}

\section{Introducción}

La ampliación y la profundización constituyen el núcleo del plan de paz que los pueblos de Europa vienen aplicando con éxito desde hace 50 años. Desde la inicial Europa de los "Seis" diseñada en los tratados fundacionales de París y de Roma de 1952 y 1957 respectivamente; las adhesiones del Reino Unido Irlanda y Dinamarca en 1973; de Grecia en 1981; de España y Portugal en 1986; de la Alemania Oriental en 1990; y finalmente de Suecia, Finlandia y Austria en 1995 han ido contribuyendo a perfilar la actual Unión Europea como ente jurídico político supra nacional y sui generis, que se ha desarrollado a lo largo de 50 años y es aún plenamente dinámico.

El actual proceso de ampliación al Este y Sur del continente al que se enfrenta la Unión Europea representa un reto novedoso en su historia, debido a la ausencia de precedentes por lo que se refiere al alcance y diversidad, al elevado número de países candidatos, a la extensa área que cubren y al importante volumen de población que la habitan.

\section{Principales procesos político-jurídicos de preparación} para las ampliaciones del siglo XXI

Poco después de la caída del muro de Berlín en 1989, los países de la antigua Europa del Este rompieron con el sistema de socialismo real vigente, sin apenas violencia, excepto en Rumania y en particular en $\mathrm{Yu}$ goslavia. A partir de 1990 entraron en una nueva etapa, en un proceso de transición único, con el objetivo de consolidar el sistema democrático parlamentario en el aspecto político, recuperando la "independencia y soberanía", enterrándose la doctrina de la soberanía limitada y construyendo un Estado de derecho conforme al modelo occidental. En lo económico y social, este proceso implica introducir un cambio de estructuras para 
seguir las pautas de la economía de mercado, así como para que la sociedad civil recupere su protagonismo e identidad sociocultural.

La transición en los países del Este de Europa que sigue a las llamadas "revoluciones gloriosas", y que se produce en el marco del proceso de preadhesión a la Unión Europea tiene un carácter no comparable con otros procesos de cambio. ${ }^{1}$

A partir de 1989, la Comunidad Europea entabló relaciones diplomáticas con los países del Centro y del Este europeo (PECOs) antiguos satélites soviéticos, apoyando su proceso de transición. Pronto se eliminaron los contingentes o cuotas a las importaciones de algunos productos, se amplió el sistema generalizado de preferencias y se celebraron acuerdos comerciales y de cooperación con Bulgaria, la antigua Checoslovaquia, Eslovenia, Estonia, Hungría, Letonia, Lituania, Polonia y Rumania. Desde 1989, el Programa PHARE creado por la Comisión Europa a instancias del G-7 (considerando que correspondía a Europa el liderazgo y la responsabilidad en la reunificación del continente) prestó su apoyo financiero a los países de Europa central y oriental para reformar y remozar sus economías. PHARE se convirtió en el programa de ayudas más importante del mundo, prestatario de conocimientos técnicos y de apoyo económico.

Durante la década de los años noventa, la Comunidad Europea y sus Estados miembros celebraron diferentes acuerdos de asociación conocidos como "Acuerdos Europeos" con diez países de Europa Central. ${ }^{2}$ Los primeros de ellos fueron firmados con Hungría y con Polonia en diciembre de 1991, entrando en vigor en 1994; el más reciente es el acordado con Eslovenia en junio de 1996, que entró en vigor en febrero de 1998. Estos acuerdos sentaron las bases jurídicas de las futuras relaciones bilaterales y han sido completados por protocolos sectoriales, por ejemplo los relativos al establecimiento de un marco para la participación en programas europeos, los Acuerdos Europeos sobre Evaluación de Conformidad y la Aceptación de Productos Industriales, etcétera. ${ }^{3}$ Los Acuerdos Euro-

1 Martín de la Guardia, R. M. y Pérez Sánchez, G. A., Los países de la antigua Europa del Este y España ante la ampliación de la Unión Europea, Valladolid, Universidad de Valladolid, 2001, pp. 20 y ss.

2 Estos acuerdos establecen sistemas administrativos de vigilancia de su desarrollo, entre los que destaca el Consejo de Asociación que a su vez aprueba decisiones sobre la participación en los programas, agencias comunitarias, comités, etcétera.

3 Para un repaso sobre estos acuerdos, véase "Los acuerdos comerciales preparan la ampliación de la Unión Europea”, Empresa Europa, núm. 4, julio-septiembre de 2001, pp. 6 y 7. 
peos se unían a otros acuerdos de asociación preexistentes con Turquía desde 1963 (en vigor desde diciembre de 1994), Malta desde 1970 (vigente desde abril de 1971) y Chipre desde 1972 (vigente desde 1973); y sus contenidos políticos, jurídicos y económicos permitieron incrementar considerablemente las relaciones comerciales entre la Unión Europea y los países de Europa Central y Oriental. En ellos ya se ponía de manifiesto la voluntad de ingresar en la Unión Europea, deseo que se haría más explícito en las solicitudes de adhesión de estos países, que se fueron sucediendo desde 1987 en el caso de Turquía, hasta 1996 en el caso de Eslovenia.

La evolución del proceso de preparación para las próximas adhesiones puede seguirse a través de los impulsos políticos reflejados en las conclusiones de las distintas presidencias del Consejo Europeo, y en ciertas comunicaciones de la Comisión Europea:

El Consejo Europeo de Copenhague de 1993 abrió definitivamente el camino de la incorporación, fijando los criterios fundamentales para la adhesión: estabilidad de las instituciones garantes de la democracia, Estado de derecho, respeto de los derechos humanos y protección de las minorías, economía de mercado, capacidad de soportar la presión de las fuerzas de la competencia y del mercado, y de asumir las obligaciones que requiere la calidad de Estado miembro. El Consejo Europeo de Essen, de finales de 1994, definió una estrategia de preadhesión basada en la aplicación de los Acuerdos Europeos, el programa PHARE de asistencia financiera, y un diálogo estructurado que agruparía a todos los Estados miembros. El Consejo Europeo de Madrid de diciembre de 1995 volvió a incidir en las condiciones de la ampliación al Este, y en particular en el requisito de la adaptación de estructuras administrativas y la transposición del derecho comunitario a través de instituciones estables, esencial para afianzar la confianza en la Unión Europea. En ese mismo Consejo Europeo de Madrid de 1995 se pidió formalmente a la comisión la presentación de una evaluación de las solicitudes de adhesión.

Como consecuencia de estos mandatos, la comisión presentó en julio de 1997 su comunicación conocida como Agenda 2000, que no sólo se refería a la situación de los países candidatos a la adhesión, sino que esbozó las líneas del futuro desarrollo de la unión, particularmente en lo referente a la financiación, recursos propios y a los dos sectores que absorben la mayor parte del presupuesto comunitario: la agricultura y la política estructural. Como anexos a la Agenda 2000, se publicaron los dictámenes sobre las solicitudes de adhesión de cada uno de los países candidatos. En 
este documento se recomendó la iniciación de negociaciones con seis países: República Checa, Eslovenia, Estonia, Hungría, Polonia y Chipre.

El Consejo Europeo de Luxemburgo de diciembre de 1997 lanzó el proceso que posibilitaría la ampliación de trece países: Bulgaria, Chipre, La República Checa, la República Eslovaca, Eslovenia, Estonia, Hungría, Letonia, Lituania, Malta, Polonia, Rumania y Turquía, si bien mantuvo el sistema propuesto por la comisión, recomendando la inicial apertura de negociaciones con seis de los países candidatos. Además, decidió que dicho proceso de ampliación comprendería:

La Conferencia Europea, marco multilateral que reuniría a los diez países de Europa Central y Oriental, a Chipre y a Turquía; cuya primera reunión tuvo lugar en Londres el 12 de marzo de 1998:

a) El proceso de adhesión, que comenzó el 30 de marzo de 1998, que agrupó a los diez países de Europa Central y a Chipre.

b) Las negociaciones de adhesión que comenzaron el 31 de marzo de 1998, con la participación de seis países según recomendación de la Comisión Europea: Chipre, la República Checa, Eslovenia, Estonia, Hungría y Polonia.

El proceso de preadhesión, y las negociaciones en el mismo sentido, no sólo se orientaban al cumplimiento por parte de los candidatos de los requisitos jurídicos y económicos establecidos por la Comunidad Europea, sino que ya desde 1997 contempla el deseo de su participación en programas y agencias de la comunidad, para permitir su adquisición de experiencia en la gestión y desarrollo institucional y político. Esta participación supone que los países candidatos adquieren responsabilidad sobre la gestión, participación e incluso sobre la financiación de tales programas y agencias, conforme a programas negociados al efecto, ${ }^{4}$ y abarca también la entrada en los comités de ejecución de tales programas. ${ }^{5}$

4 El régimen de participación ha sido modificado a partir de diciembre de 2001, a raíz de la propuesta de la comisión relativa a la posición de la comunidad en el Consejo de Asociación acerca de la participación en programas comunitarios (COM 2000, 725 final). El proceso consiste, en primer lugar, en un acto unilateral de la comunidad, habitualmente una decisión del consejo que "abre los programas" a la participación de los países candidatos. Después, cada uno de éstos debe realizar una solicitud. Hasta 2001, la participación era objeto de una negociación bilateral en el seno de los consejos de los Acuerdos de Adhesión, y las bases pasan a formar parte de tales acuerdos o bien de protocolos a los mismos. A partir de diciembre de 2001 se han ido aprobando 13 acuerdos marco, uno con cada candidato, en los que se establecen las condiciones generales de participación. Cada uno de tales acuerdos será completado por memoranda, concluidos por la comisión y las diversas autoridades gubernamentales de los candidatos, en relación con cada programa concreto (participación financiera, calendarios, condiciones específicas, etcétera.)

5 Comisión Europea, "Comunicación de la comisión al consejo, participación de los países 
La Comisión Europea sigue presentando anualmente dictámenes sobre los avances realizados por cada uno de los países candidatos en su camino hacia la adhesión para comprobar el seguimiento del acervo comunitario (screening), que a efectos de este proceso se agrupó en 35 capítulos. En 1999 varió la postura que había fijado en la agenda 2000 (que consistía en agrupar a los países candidatos en dos bloques) y recomendó la apertura de negociaciones con el resto de los candidatos, incluida Malta. ${ }^{6}$ Este sistema fue aceptado políticamente de modo que el Consejo Europeo reunido en Helsinki en diciembre de 1999 decidió emprender formalmente las negociaciones de adhesión con otros seis países candidatos: Bulgaria, la República Eslovaca, Letonia, Lituania, Malta y Rumania. Estas se iniciaron formalmente el 15 de febrero de 2000. Por otra parte, el Consejo Europeo de Helsinki confirmó la aplicación de los mismos criterios de adhesión para Turquía.

En el Consejo Europeo de Niza de diciembre de 2000, en el seno de la Conferencia de Representantes de los Estados Miembros, se adoptó el acuerdo para reformar los tratados fundacionales de París, Roma y Maastricht con el nuevo Tratado de Niza firmado en febrero de 2001 (aún en proceso de ratificación). Las negociaciones de la futura ampliación confluyeron con otro proceso interno que se ha venido desarrollando en el seno de la Unión Europea, que ya quedaba anunciado en el Tratado de Maastricht de 1992 y subrayado con el Tratado de Amsterdam de 1997: "la necesidad de adaptar las instituciones para permitir más ampliaciones".

Los consejos europeos de Estocolmo de marzo de 2001 y de Gotemburgo de junio de 2001 continuaron el análisis detallado de los informes de la comisión sobre los requisitos de los países candidatos para la ampliación, en el marco de los resultados y debates del "Debate sobre el futuro de Europa", enraizado en la Declaración 23 del Tratado de Niza. El Consejo Europeo de Laeken, de diciembre de 2001, establece expresamente en su artículo 8o. que "La Unión Europea está decidida a llevar a buen término las negociaciones de adhesión para finales de 2002 con los países candidatos que estén preparados, con el fin de que puedan participar en calidad de miembros en las elecciones al Parlamento Europeo de

candidatos en los programas, agencias y comités comunitarios", COM (1999), 710 final, Bruselas, 20 de diciembre de 1999. Esta comunicación fue modificada en lo referente a la participación en programas por el ya citado COM (2000) 725 final.

6 Malta había congelado su solicitud de adhesión en 1996 y decidió seguir adelante con el proceso en octubre de 1998. 
2004". En la misma reunión se aprobó la declaración sobre "El futuro de Europa" también conocida como "Declaración de Laeken", en la que se recuerda que la Unión Europea es ante todo el resultado de un proceso aún abierto que tiene ante sí muchos retos, como el mejor reparto y definición de competencias de la unión, la simplificación, democracia y trasparencia. En ese mismo consejo quedó convocada una convención (presidida por el polémico Giscard d'Estaing, y vicepresidida por Amato y Deahene) que prepare la futura conferencia intergubernamental. ${ }^{7}$

Pese a las declaraciones de buena voluntad, y a que una gran parte de los capítulos de negociaciones sobre acervo ya han sido concluidos, aún quedan "flecos" en las negociaciones. Se trata de cuestiones particularmente complicadas, algunas de las cuales ya hubieran debido cerrarse. En el más reciente documento estratégico de la comisión de octubre de 2001, quedan patentes algunos datos: Chipre es el país que más capítulos ha cerrado (23) Además es el Estado con mejor situación económica (un 83\% del PIB de la media comunitaria), y cuenta con un paro muy bajo $(3,4 \%)$. Su principal escollo es su división territorial tras la invasión de Turquía del norte de la isla. Hungría se sitúa en segundo lugar por capítulos cerrados y cuenta con una situación económica estable donde destaca su tasa de paro de un 6,4\%. También en primera línea se encuentra la República Checa que ya desde 1997 cumplía los requisitos políticos, y que está reformando muy rápidamente sus sistemas judiciales y legales; así como Eslovenia que ha progresado considerablemente en el proceso de refuerzo y profundización de la estabilidad de las instituciones.

En la zona "media" se encuentran países como Eslovaquia, país que tiene la tasa más alta de paro $(18,6 \%)$ y un déficit público del $6,7 \%$, o Estonia. Por lo que respecta a Polonia, es generalmente aceptado que su adhesión se realizará necesariamente en primer lugar, aunque está sufriendo dificultades. Cuenta con una población compuesta en un $20 \%$ por agricultores, y es precisamente en el terreno de la agricultura, fondos regionales y liberalización de la adquisición de tierras por parte de extranjeros donde tiene más dificultades. ${ }^{8}$ Los aspectos relacionados con la agri-

7 Raux, J., “Après Nice: Pour une approche structurante de la constitutionalisation de l'Union Européenne", citado en Vilá Costa, B., "El horizonte institucional de la UE tras la conferencia intergubernamental (de Biarritz a Niza)", Cuaderns de Treball, núm. 38, noviembre de 2001, pp. 7 a 24.

8 La Constitución polaca exige una consulta popular vinculante sobre el ingreso en la Unión Europea. Los sondeos daban un apoyo de un 52\% antes del paquete de propuestas sobre agricultura a comienzos del 2002, por las que la comisión propone la introducción progresiva de ayudas directas a los agricultores durante un periodo de 10 años a partir de 2004. 
cultura, así como con la cohesión económica y social reforzada mediante fondos estructurales, se encuentran entre los últimos que es necesario "cerrar" de modo previo a las adhesiones. Constituyen aspectos que ya fueron contemplados en la agenda 2000, y que resultan críticos en la actual Europea de 15 miembros, incluso sin el elemento añadido de la ampliación.

El Consejo Europeo de Barcelona de 15 y 16 de marzo de 2002 acogió el Informe del secretario general del consejo con vistas a la reforma del consejo ante la ampliación. Y el Consejo Europeo de Sevilla debía haber adoptado decisiones relativas a estas reformas de forma previa a la ampliación, pero la circunstancias que rodean ciertas negociaciones, en particular las relativas al capítulo agrícola, no lo han hecho posible.

\section{El proceso de adaptación interna de la Unión}

Europea: las instituciones de la Unión Europea

y las ampliaciones del siglo XXI

La Constitución de la inicial "Europa de los seis" reunía a un reducido grupo de países relativamente homogéneos y que presentaban un equilibrio entre "países grandes" (Alemania, Francia, e Italia) y países pequeños (Holanda, Bélgica y Luxemburgo). ${ }^{9}$ Esta configuración inicial hacía posible el funcionamiento comunitario a través de unas instituciones, ideadas en aquel momento para la adopción supranacional de decisiones, según el criterio de la unanimidad. La regla de la unanimidad ha ido abandonándose progresivamente con las nuevas reformas de los tratados fundacionales, hasta llegar al Tratado de Niza, en proceso de ratificación en la actualidad, en el que queda reducida a muy pocos asuntos como la fiscalidad o la seguridad social. La adopción de decisiones por mayoría permite agilizar la toma de decisiones, lo que se hace más importante a medida que la unión se amplía.

Las instituciones más implicadas en la toma de decisiones comunitaria son la comisión, el consejo y el Parlamento europeos. En ellas, el número de miembros y su composición fueron ideados originariamente teniendo en cuenta las dimensiones geográficas y la población de cada uno de los países integrantes. En todo momento se intentó lograr el equilibrio entre países "grandes" y "pequeños", así como entre "territorio" y "po-

9 Pérez Carrillo, E. F., “El Tratado de Niza entre la consolidación de la UE y el debate sobre el futuro de Europa”, Anuario Mexicano de Derecho Internacional, vol. II, 2002. 
blación". Por ello, y para evitar que los países "pequeños" se sintiesen "materialmente anexionados", en 1957 se decidió "sobredimensionar" los votos en el consejo. Esta lógica se mantuvo a lo largo de las diversas ampliaciones que supusieron la entrada de un país "grande" (Reino Unido), uno intermedio (España) y siete pequeños (Dinamarca, Irlanda, Grecia, Portugal, Austria, Suecia y Finlandia). En consecuencia, a medida que se iban produciendo tales adhesiones, la importancia relativa de los "países grandes" se iba reduciendo. ${ }^{10}$

Esta dinámica condujo a un considerable incremento en el tamaño de las instituciones, que de mantenerse podría conducir al colapso y la ineficacia. Por eso, ya desde el Tratado de Maastrich de 1992 se contempló la necesidad de revisar los mecanismos internos de adopción de decisiones y de reformar las instituciones. El artículo N-2 del Tratado de Maastricht condujo a la reforma de Amsterdam en 1997, que no logró el consenso de fondo, pero que introdujo un Protocolo sobre "Las instituciones y la Ampliación", y contempló además la convocatoria de una nueva conferencia intergubernamental en el 2000 para acometer la transformación institucional. La Conferencia fue convocada en el Consejo Europeo de junio de Colonia 1999. Se reunió a lo largo del año 2000 durante las presidencias portuguesa y francesa, y presentó el borrador definitivo de Tratado en Niza en diciembre de 2000. En este texto se refleja el reparto de vo-tos en el seno de las instituciones, teniendo en cuenta el proceso de ampliación. Es decir que ya se valoran los elementos de población y territorio de los países candidatos del Este y del Sur del continente, con vistas a la adecuación de las instituciones europeas a las futuras ampliaciones. De no haberse introducido estos cambios, y de haberse seguido el sistema de reparto de escaños y votos anterior, las ampliaciones previstas acentuarían el desequilibrio, ya que la enorme mayoría de los países candidatos responden a la clasificación en el grupo de "países pequeños", con la excepción de Polonia que es un país "intermedio".

Recordemos algunos de los cambios institucionales introducidos en Niza: ${ }^{11}$

10 Best, E., “The Treaty of Nice: Not Beautiful but it'll Do", Eipascope, núm. 1, pp. 2-9, esp. p. 3.

11 Sobre las reformas introducidas por el aún no ratificado Tratado de Niza, ya realizamos una exposición en este mismo foro (que ahora reproducimos de forma resumida), véase Pérez Carrillo, E. F., op. cit., nota 9. 
Las modificaciones del Consejo y los votos asignados a los miembros de la Unión y a los candidatos a serlo, fueron objeto del mayor debate, en torno al ajuste de la influencia relativa de los Estados ante la perspectiva de una Unión ampliada, de un intento de re-equilibrar las diferencias demográficas y el poder de decisión y de garantizar la existencia de un ejecutivo eficiente, incluso ante la Ampliación sin prevista. De haberse mantenido el sistema vigente se abriría la vía para que las decisiones del Consejo estuviesen respaldadas por un porcentaje reducido de la población europea (en torno a un 50\%). El Tratado de Niza introduce modificaciones en el sistema de ponderación que no entran en vigor hasta el 1 de enero de 2005.

El Tratado de Niza introduce una nueva reponderación y una triple mayoría: A partir de 1o. de enero de 2005 el numero de votos en el Consejo oscilará entre los 29 de cada uno de los Estados más poblados a los 4 votos ponderados asignados a Luxemburgo. La fijación definitiva de porcentajes y umbrales de mayoría dependerá del ritmo de las ampliaciones, por lo tanto se tendrán que ir adaptando proporcionalmente a medida que entren nuevos socios. En un principio, la nueva la mayoría cualificada quedará fijada en 169 votos de los 237, frente al sistema actual de 62 sobre 87. En una Europa de 27 miembros la mayoría cualificada será de 258 votos sobre un total de 345, lo que imposibilita la Unión de tres "grandes" que por sí solos puedan bloquear la adopción de decisiones. Además cualquier Estado puede solicitar a través de su representante en el Consejo, que se compruebe que la mayoría cualificada representa al menos un $62 \%$ del total de la población. Las modificaciones que afectan a esta institución aún no han terminado. Así se ha puesto de manifiesto en el documento presentado por el secretario general del consejo, Javier Solana, al Consejo Europeo de Barcelona de 15 de marzo de 2002, donde se realizan propuestas tendentes a la restructuración del Consejo y a la modificación de sus métodos de trabajo para hacerlos más operativos y prácticos en una Unión ampliada. ${ }^{12}$

Por lo que respecta a la Comisión, el Tratado de Amsterdam de 1997 preveía que las modificaciones en su seno quedarían vinculadas a las modificaciones que se produjesen en el Consejo. Se partía de la necesidad de poner un límite en el número de Comisarios para evitar que esta Institución se convierta en una "asamblea de delegados nacionales". En Niza, los Quince decidieron que a partir del 1 de enero de 2005 los Estados que en la actualidad tienen dos Comisarios (Alemania, Francia, Reino Unido, Italia y España) perderán uno. Cada nuevo país miembro contará con un Comisario. Cuando el colegio alcance los 27 miembros, los Estados tendrán que 
decidir por unanimidad cual deba ser el número de miembros de la Comisión, que será inferior al de Estados.

El Parlamento Europeo es la Institución que menos cambios estructurales ha sufrido. En Amsterdam se había acordado que el número máximo de Europarlamentarios sería de 700, cifra superada ahora para pasar a 732 en el Tratado de Niza, en gran medida a causa de las negociaciones respecto del equilibrio franco-alemán. El resto los “grandes” pasan a tener 72 representantes.

A pesar del "no irlandés" a la ratificación que se produjo en el referéndum de junio de 2001, se están buscando soluciones para la rápida entrada en vigor del Tratado de Niza, con el fin de permitir las nuevas adhesiones desde el año 2003. ${ }^{13}$

\section{COHESIÓN EN UNA EUROPA AMPLIADA}

Los tratados de París y de Roma no hacían referencia expresa a la cohesión económica y social, pese a que ya reflejaban una voluntad política que había sido expresada por sus principales inspiradores Jean Monnet y Robert Schuman "la loi de la solidarité des peuples s'impose à la consciencie comtemporaine" (Schuman 1963), que se tradujo en la "solidaridad entre los pueblos de Europa" del preámbulo de los tratados fundacionales.

La necesidad de lograr una auténtica cohesión económica y social fue evidenciándose a medida que se avanzaba en la profundización del mercado interior. La libre circulación de mercancías y de factores de producción (trabajadores, servicios y capitales), beneficiosa para el desarrollo del continente, repercutía no obstante en el incremento de las disparidades regionales. La política regional y de cohesión fue elevada al rango de derecho originario en el Acta Única Europea de $1986,{ }^{14}$ en gran medida como consecuencia del ingreso en la comunidad de tres países mediterráneos: Grecia, Portugal y España, que presentaban unas rentas nacionales bastante inferiores a la media comunitaria. La entrada de los Länder del Este alemán y de ciertas zonas especiales de Suecia y Finlandia, junto con

13 Best, E., op. cit., nota 10, pp. 2-9, esp. p. 6.

14 La política regional constituye una de las consecuencias de la voluntad política de contribuir a la más plena cohesión económica y social, aunque no sea la única. El transporte, las ayudas estructurales a la agricultura y el desarrollo rural, el medio ambiente y otras políticas también forman parte del objetivo de cohesión. 
la propia dinámica de la evolución europea fueron modelando el sistema de ayudas regionales.

En la actualidad, los vigentes reglamentos estructurales aprobados en 1999 replantean la política de cohesión en el marco del desarrollo de la Agenda 2000 de 1997, es decir como punto de equilibrio entre la financiación del equilibrio estructural interno y las previstas ampliaciones. Los fondos estructurales constituyen instrumentos de cohesión de cara a la superación de desequilibrios en la Europa de 15 miembros, y previsiblemente continuarán siéndolo de cara a la ampliación al Centro y Este.

La ampliación de la unión a 27 Estados supondrá un incremento territorial en torno a un $34 \%$ y de un $28 \%$ en términos de población, pero sólo de un 5\% en relación con el PIB. En consecuencia, el PIB por habitante disminuirá en un $18 \%$. Por otra parte, de los 105 millones de habitantes de la unión de 27 Estados, más de 98 millones habitarán en regiones cuyo PIB será inferior al $75 \%$ de la media. ${ }^{15}$ Unas transformaciones tan radicales imponen una auténtica reforma de la política de cohesión y de su principal instrumento, los fondos estructurales, a partir de 2006. No se trata únicamente de saber quien tendrá derecho a que porcentaje del reparto de fondos estructurales, sino de definir cuáles son los resultados deseables. Es decir, estamos ante una necesaria redefinición de la política estructural comunitaria.

Recientes estudios de la comisión muestran que si la ampliación a 27 miembros se produjese en el año 2002, la Unión Europea quedaría conformada en tres grupos de países en lo que se refiere a su nivel de desarrollo económico: ${ }^{16}$

- El primero estaría formado por la mayoría de países actualmente candidatos, con un nivel de vida equivalente al $41 \%$ de la media comunitaria.

- El segundo quedaría constituido por tres países candidatos: Chipre, Eslovenia y la República Checa, y tres Estados que en la actualidad son ya miembros de la unión: Grecia, España y Portugal, con un nivel de vida equivalente al $87 \%$ de la media.

15 Actes du Deuxième Forum Européen sur la Cohesion, Bruselas, 21-22 de mayo de 2001. Conferencia de A. Diamantopoloupou, Bruselas, 2001, pp. 69-72.

16 Dirección General de Política Regional, Comisión Europea, "Aprobación del informe intermedio sobre la cohesión”, Inforegio News, núm. 95, febrero de 2002, y corrección de errores, en número 96 de marzo de 2002. 
- El último grupo sería el de los doce Estados miembros restantes situados muy por encima de esas medias.

De estos datos se deduce que la ampliación no sólo tendrá grandes implicaciones para Europa del Este, sino también para los actuales 15 miembros, y sobre todo para la puesta en marcha de una política regional y de cohesión. El bajo nivel económico de los nuevos socios (ninguno de los 13 países PECO alcanzaron en 1998 el 50\% del PIB de la media comunitaria, y sólo tres lo han superado en la actualidad) supone un reto para la capacidad y la voluntad política de la Europa de los 15, que para alcanzar el desarrollo equilibrado y cohesionado debe apoyar el proceso de ampliación con fuertes ayudas financieras.

La entrada de un gran número de Estados miembros con su diversidad de lenguas, cultura, religiones, educación y regímenes de protección social, pone de manifiesto que la cohesión plantea problemas que superan con creces la cuestión económica y que ahora más que nunca se refuerza la necesidad de contar con órganos e instrumentos de adopción de decisiones donde la mayoría democrática sea combinada con la protección de los intereses de Estados, regiones y diversas minorías. El desarrollo cohesionado, equilibrado y duradero requiere hacer frente a los muy diversos factores que afectan a la sociedad y a la economía. ${ }^{17}$ Destaquemos algunos:

- La fase de transición hacia una economía de mercado ha desintegrado el modelo social de los países candidatos en aspectos tan importantes como la educación, el mercado de trabajo, la participación de la mujer o la inmigración, sin que al mismo tiempo se hayan establecido estructuras necesarias para establecer el modelo social de la unión, uno de los atractivos más importantes para que estos países pretendan su integración en la Comunidad Europea. Pues bien, no será posible contribuir a eliminar las disparidades de riqueza y de oportunidades nacionales regionales o locales que ya existen entre estos países si la unión no define un modelo adecuado de desarrollo económico.

17 Junto a los componentes sociales y geográficos de la cohesión, debemos tomar también en cuenta a la economía para el diseño de las futuras políticas. Precisamente, la Cumbre de Lisboa de marzo de 2000 subrayó la importancia de aprovechar la interdependencia entre la política económica y la política social, y resaltó que la cohesión europea se apoye en las vinculaciones que se establezcan entre ambas. 
- En un plano individual, las profundas modificaciones en el régimen de empleo y de estilo de vida hacen predecible que se produzcan fuertes migraciones. Por ello uno de los retos relacionados con la esfera social y de cohesión es el desarrollo y fortalecimiento de los sistemas de protección social de los países candidatos, que constituyen requisitos previos para la estabilidad política de toda la unión. La mayoría de los expertos están de acuerdo en que si los sistemas sociales funcionan, los países candidatos no representan una amenaza para los mercados laborales occidentales. Por el contrario permitirán equilibrar las capacitaciones técnicas de la población europea en las distintas regiones.

- La formulación de una política de cohesión del futuro parte de poner en cuestión si el actual sistema de protección social es mantenible. En este sentido se admite de forma general que el éxito económico en el futuro dependerá más que hasta ahora de los recursos y competencias de la población europea. Dada la existente movilidad del capital y de la técnica, la inversión en recursos humanos es lo que marca la diferencia de la competitividad y la cohesión. Por ello, desde el punto de vista de la economía, los fondos estructurales deberán servir a medio plazo para realizar inversiones tendentes a la creación de estructuras sociales en los países candidatos. El desmembramiento de la Unión Soviética ha producido que muchos de los mercados del Este hayan sido sustituidos por los occidentales, lo cual ha repercutido negativamente en la creación de un tejido empresarial sólido y autóctono en los países candidatos. Esta tendencia contribuye a la disparidad económica y social y debe ser corregida. Por eso será necesario apoyar la consolidación de los factores sociales y económicos que más dinamizan la economía y el empleo. En concreto será esencial promover la creación de pequeñas y medianas empresas (PYMES) para desarrollar el sector de los servicios y para incrementar la productividad y competitividad de las antiguas unidades productivas. Por otra parte, la inversión extranjera directa puede tener un papel fundamental para la mejora de la economía y su reestructuración. Aunque su contribución al empleo sea baja, puede incrementar el nivel de intercambios comerciales y servir de estímulo a los competidores locales.

- Una transición rápida requiere un sistema educativo de ciencia y tecnología desarrollado y competitivo. Las infraestructuras técnicas 
y educativas constituyen precondiciones esenciales para el funcionamiento de la economía.

- Existen deficiencias importantes en los sistemas de comunicaciones, transportes y energía en los países candidatos. Su liberalización, modernización y actualización hasta ponerse al nivel de la Europa de los 15 constituirá una labor muy importante en la próxima década.

Si la comunidad desea continuar haciendo frente a las ayudas a las regiones menos favorecidas o que sufren un menor desarrollo estructural (tanto respecto de las actuales como de las que se adherirán próximamente), debe jerarquizar claramente sus prioridades y centrarse en objetivos específicos, ya que las posibilidades de incremento del presupuesto comunitario son limitadas. Sobre esta base, los fondos estructurales del futuro deberán servir para hacer frente a cinco grandes desafíos, cada uno de los cuales deberá tenerse muy presente en la formulación de las políticas estructurales y de cohesión a partir del año 2006:

- La mundialización de las economías.

- La revolución de la información y de la comunicación.

- La modernización del sistema económico y de los sistemas de protección social, teniendo en cuenta las nuevas realidades relativas al lugar ocupado por la mujer en la sociedad y la demografía, el desarrollo sostenible y duradero.

- La importancia de lograr el éxito en la ampliación.

Si se mantiene el actual sistema de cálculo de regiones elegibles para obtener la ayuda de los fondos comunitarios, la mayoría de las actuales regiones beneficiadas perderían automáticamente este derecho, pese a que la ampliación por sí misma no solucionará las actuales deficiencias y disparidades que afectan de forma especial a ciertas zonas periféricas, rurales y urbanas. La transición para las regiones menos desarrolladas en una Europa de 15 miembros puede ser muy dura y puede crear tensiones sociales y económicas difíciles de solucionar. Por lo tanto, junto a la asistencia a los países del Centro y del Este será preciso mantener una reserva de seguridad a favor de los actuales miembros, ya sea a través de la redefinición de los criterios para la clasificación de las zonas objetivo 1, de la puesta en marcha de nuevas medidas de cohesión inter regional, la introducción de nuevos criterios horizontales o el establecimiento de medidas transitorias en materia de financiación. 
La jerarquización de los objetivos prioritarios en materia de política de cohesión hará necesario adoptar decisiones difíciles, por una parte en lo relativo al montante del presupuesto comunitario y por otra en lo relativo a la concentración de inversiones donde sean más necesarias. Aún así el equilibrio de desarrollo entre Europa oriental y occidental tardará mucho tiempo en conseguirse. A pesar de ello, muchas zonas y regiones que actualmente forman parte de la unión continuarán necesitando del apoyo de los fondos para eliminar sus propios desequilibrios. En este sentido se hará preciso concentrar las ayudas en el Este de Europa sin afectar al desarrollo de las regiones menos favorecidas de la actual unión.

El reto de la concentración de ayudas favoreciendo a los nuevos Estados, y al mismo tiempo manteniendo el apoyo a las regiones actualmente beneficiarias que no hayan superado sus deficiencias estructurales, puede significar que el 0,45\% del PIB comunitario destinado a la política de cohesión según el Consejo Europeo de Berlín de 1999 tal vez deba considerarse sólo como el umbral mínimo necesario para responder a las demandas de una unión ampliada. ${ }^{18}$

Sea cual fuere la opción que se adopte en la próxima fase de política estructural es muy posible que sea muy distinta de las que nos han precedido y que refleje no sólo los efectos de la ampliación sino también las exigencias de la nueva economía del conocimiento que impactará sobre las economías nacionales, regionales y locales, tanto en los actuales países miembros como en los actualmente candidatos a la adhesión.

\section{FINANCIACIÓN DE LA PREADHESIÓN}

La ampliación de la Unión Europea hacia los países de Europa Central y Oriental (PECO) es un reto importante para la cohesión dado el gran número de países candidatos y las grandes disparidades que se observan. A pesar de los importantes esfuerzos efectuados por los candidatos, su integración en las estructuras y programas comunitarios existentes será una tarea compleja. La cuantía de asistencia financiera a favor de los países candidatos debe ser políticamente posible para los Estados miembros y suficiente para crear un impacto visible. La cuantía de ayudas re-

18 Las conclusiones del Consejo Europeo de Berlín fueron desarrolladas en otros importantes textos relativos a la financiación de la unión. Destaca el informe de la comisión sobre el funcionamiento de los recursos propios, redactado en el marco de la agenda 2000, y sobre la base del documento COM (1998) 560 final (suplemento 2/98 del Boletín de la Unión Europea). 
gionales, estructurales y de cohesión previstas para la integración de los países PECO, es muy superior a la que fue facilitada por otros programas de recuperación para Europa como el Plan Marshall. En la actualidad la suma de ayudas en programas regionales, estructurales y de cohesión de la Unión Europea representa un sexto del peso relativo de gasto del presupuesto de Estados Unidos de América. Además, frente al Plan Marshall que tuvo una duración de cuatro años, no hay una fecha límite definitiva para el término de la ayuda europea. La estrategia de preadhesión para el periodo 2000-2006 consiste en aplicar una estrategia global de preadhesión para preparar a estos países para la adhesión a la Unión Europea, agrupar las distintas formas de ayudas ofrecidas por la unión en un marco único y familiarizar a los países candidatos con los procedimientos y las políticas de la unión para permitirles participar en los programas y agencias comunitarios, ayudándoles a cumplir el acervo comunitario. Las ayudas financieras se canalizan fundamentalmente a través de tres instrumentos: PHARE, para la consolidación de las instituciones de los PECO, su participación en programas comunitarios, el desarrollo regional y social, la reestructuración industrial y el desarrollo de las PYME; SAPARD, para la modernización de la agricultura y el desarrollo rural, e ISPA o instrumento estructural de preadhesión para el desarrollo de infraestructuras de transporte y de protección del medio ambiente.

Desde el 2000, la unión duplicó la ayuda que concede en el marco de la preadhesión que ha pasado a ser de más de 3 mil millones de euros al año (véase el cuadro I en la siguiente página). Conforme los países candidatos se vayan adhiriendo, se beneficiarán de una asistencia especial de ampliación.

\section{El programa PHARE}

La estrategia de preadhesión consiste en una combinación de un abanico de prioridades con apoyo financiero, acuerdos de asociación, participación en programas y agencias comunitarias y preparación de las negociaciones mediante un examen analítico del acervo. El Programa PHARE de asistencia a Polonia y Hungría iniciado en 1989 se amplió tras la invitación del Consejo de Copenhague de 1993 a todos los países del Centro y Este de Europa y se incrementó considerablemente su infraestructura. Sin embargo, PHARE sólo puso el acento a la preadhesión en 1997 en respuesta al lanzamiento del proceso actual de ampliación en el Consejo Europeo de Luxemburgo. 
CUADRO I: LA ASISTENCIA FINANCIERA DE PREADHESIÓN APORTADA POR LOS TRES INSTRUMENTOS PARA EL PERIODO 2000-2006 (EN MILLONES DE EUROS Y CON PRECIOS DE 1999)

\begin{tabular}{|l|r|r|r|r|r|r|r|r|}
\hline Año & 2000 & 2001 & 2002 & 2003 & 2004 & 2005 & 2006 & Total \\
\hline PHARE & 1560 & 1560 & 1560 & 1560 & 1560 & 1560 & 1560 & 10920 \\
\hline SAPARD & 520 & 520 & 520 & 520 & 520 & 520 & 520 & 3640 \\
\hline ISPA & 1040 & 1040 & 1040 & 1040 & 1040 & 1040 & 1040 & 7280 \\
\hline Total & 3120 & 3120 & 3120 & 3120 & 3120 & 3120 & 3120 & 2184 \\
\hline
\end{tabular}

FuENTE: Comisión Europea.

Con su presupuesto anual de 1.560 millones de euros cofinancia la consolidación de las instituciones con inversiones en las infraestructuras reglamentarias para la aplicación del acervo. Mediante un programa de "emparejamiento", los funcionarios de los Estados miembros ayudan a sus homólogos a preparar el ingreso. También se ha integrado la gestión de PHARE en la estructura de gobierno de los países candidatos por medio de la creación del Fondo Nacional, y de un pequeño número de agencias encargadas de poner en práctica las medidas. Gracias a la ayuda para la adaptación al acervo comunitario, en la actualidad la mayoría de los países candidatos disponen de una base jurídica sólida en lo que respecta al mercado interior, pese a que ciertas partes de la legislación tardarán en adoptarse, como la integración de los servicios públicos en la legislación relativa a la contratación pública, la aproximación de los tipos de IVA y de los impuestos específicos sobre el consumo y la aplicación eficaz del sistema comunitario de normas y certificación. Entre los sectores que muestran mayor retraso de adaptación está el audiovisual, donde muy pocos países candidatos se han ajustado a la "Directiva Televisión sin Fronteras", o el sector de la seguridad nuclear.

PHARE es un programa pasarela hacia los fondos estructurales. A través de la oficina de intercambio de informaciones sobre asistencia técnica, TAIEX posibilita disponer de expertos para asesoramiento a corto plazo. Este enfoque permite concentrar los refuerzos en los principales retos a los que deben responder los países candidatos, según los análisis periódicos o "escrutinio" que comenzó en los PECO en marzo de 1998.

En 1999 se ajustaron sus orientaciones para reflejar el lanzamiento del programa SAPARD e ISPA, reorientándose un tercio de los fondos 
PHARE a la consecución de la cohesión económica y social, a la que contribuyen también otros instrumentos.

\section{ISPA: Instrumento estructural de preadhesión, cohesión, medio ambiente y transportes}

Dentro de los instrumentos financieros de preadhesión, ISPA, por la similitud en su funcionamiento con el Fondo de Cohesión y su carácter de instrumento estructural, es el que más incide en lograr la cohesión económica y social con anterioridad a la adhesión. Su acción abarca preparar a los países candidatos a la adhesión familiarizándolos con las políticas y procedimientos de la unión, ayudándoles a cumplir normas medioambientales comunitarias y dándoles acceso a una red transeuropea de transportes más extensa. Las actuaciones en materia medio ambiental se centrarán en el suministro de agua potable, el tratamiento de aguas residuales, la gestión de residuos sólidos y la contaminación del aire. Resulta indispensable desplegar esfuerzos así como considerable ayuda técnica y financiera para que estos países alcancen rápidamente el nivel exigido de acervo comunitario en materia de medio ambiente. Por lo que respecta al transporte, la ayuda de la Unión Europea se destinará fundamentalmente a los proyectos de infraestructuras que favorezcan la movilidad sostenible, los proyectos de interés comunitario y los que permitan a los países interesados ajustarse a los objetivos de las asociaciones para la adhesión. Las redes de transportes transeuropeas se extenderán para crear buenas conexiones entre la actual Europa de los 15 y los países candidatos, así como interconexiones entre las redes nacionales y su enlace con las redes transeuropeas. Las inversiones previstas en materia de transporte en los países candidatos deberán ser muy considerables para situarles al nivel de los actuales Estados miembros. La contribución del ISPA consiste por tanto en el apoyo financiero al desarrollo de ferrocarriles, puertos y aeropuertos, teniendo en cuenta las exigencias del transporte sostenible, que utilice los recursos del presente sin poner en riesgo el futuro, y la intermodalidad. Una pequeña parte de la ayuda facilitada por este programa puede utilizarse para financiar estudios previos y de asistencia técnica, siempre que quede claramente establecida la relación entre estas medidas y los proyectos finalmente ejecutados, así como su repercusión en la calidad de los resultados globales. 
ISPA dispone para el periodo 2000-2006 de 7280 millones de euros, que representan 1.040 millones de euros anuales. La comisión ha realizado un reparto indicativo entre los Estados candidatos, en forma de bandas (véase el cuadro II en la siguiente página) para garantizar una gestión flexible, premiar los resultados a medida que se produzcan y para permitir la distribución equitativa entre proyectos medioambientales y de transportes. Entre los países destinatarios de la mayor parte de la ayuda están Polonia (entre un 30,0\% y un $37,0 \%$ ) y Rumania (entre un $20,0 \%$ y un $26,0 \%$ ), frente a Eslovenia, que recibirá los porcentajes menores (entre un 1,0\% y un 2,0\%).

Siguiendo el modelo del Fondo de Cohesión, donde la ayuda financiera se concede caso por caso, ISPA financia proyectos concretos. Cada proyecto se define como una serie económicamente indivisible de trabajos con una función técnica precisa y con objetivos definidos. Puede también financiar fases de un proyecto, esto es, fases técnica y económicamente independientes que puedan considerarse operativas como tales. También permite acometer la financiación conjunta de grupos de proyectos que respondan a tres condiciones:

a) Estar situados en la misma zona o en el mismo eje de transporte.

b) Que formen parte de un plan global para cada zona o eje.

c) Que estén supervisados por un único organismo de supervisión y de vigilancia.

Los proyectos financiados con ISPA deben hacerse teniendo en cuenta los programas nacionales sobre transporte y medio ambiente, esenciales dentro de las asociaciones de adhesión, y han de tener impacto significativo en la protección medioambiental o en la mejora de las redes de transportes, y salvo situaciones excepcionales, únicamente pueden subvencionarse los proyectos de más de 5 millones de euros. El porcentaje de cofinanciación de la Unión Europea puede llegar hasta un 75\% del gasto público o en casos excepcionales hasta un $85 \%$, según los criterios de: posibilidades de cofinanciación, rentas potenciales generadas y aplicación del principio de "quien contamina paga" establecido con carácter general en el artículo 174 del TCE.

En la actualidad ISPA, que ha cumplido su segundo aniversario, ha sido utilizado para financiar abundantes proyectos medioambientales y de transportes en todos los países candidatos, entre los que destacamos algunos: En Bulgaria se han invertido 349 millones de euros para la construcción de plantas regionales de depuración de aguas residuales y para la electrificación de la red ferroviaria; en la República Checa se han inverti- 
CUADRO II. REPARTO ANUAL DE LOS FONDOS DE PREADHESIÓN, 2000-2006 (EN MILLONES DE EUROS Y CON PRECIOS DE 1999)

\begin{tabular}{|l|r|r|r|r|r|r|}
\hline & \multirow{2}{*}{ PHARE } & \multicolumn{1}{c|}{ SAPAR } & \multicolumn{2}{|c|}{ ISPA } & \multicolumn{2}{|c|}{ Total } \\
\hline & & & Mínimo & Máximo & Mínimo & \multicolumn{1}{c|}{ Máximo } \\
\hline Bulgaria & 100,0 & 52,1 & 83,2 & 124,8 & 235,3 & 276,9 \\
\hline R. Checa & 79,0 & 22,1 & 57,2 & 83,2 & 158,3 & 184,3 \\
\hline Estonia & 24,0 & 12,1 & 20,8 & 36,4 & 56,9 & 72,5 \\
\hline Hungría & 96,0 & 38,1 & 72,8 & 104,0 & 206,9 & 238,1 \\
\hline Letonia & 30,0 & 21,8 & 36,4 & 57,2 & 88,2 & 109,0 \\
\hline Lituania & 42,0 & 29,8 & 41,6 & 62,4 & 113,4 & 134,2 \\
\hline Polonia & 398,0 & 168,7 & 312,0 & 384,8 & 878,7 & 951,5 \\
\hline Rumania & 242,0 & 150,6 & 208,0 & 270,4 & 600,6 & 663,0 \\
\hline Eslovaquia & 49,0 & 18,3 & 36,4 & 57,2 & 103,7 & 124,5 \\
\hline Eslovenia & 25,0 & 6,3 & 10,4 & 20,8 & 41,7 & 52,1 \\
\hline Total & 1085,0 & 520,0 & & 1040,0 & & 2645,0 \\
\hline
\end{tabular}

FuENTE: Comisión Europea. Presupuesto total anual de PHARE: 1.577 millones de euros.

do 171,4 millones de euros en la construcción de carreteras, plantas de tratamiento de agua potable y depuración de aguas residuales; en Estonia, 82,4 millones de euros para la construcción de carreteras, modernización de la red de alcantarillado, construcción de plantas regionales de tratamiento de residuos y asistencia técnica; en Hungría 337,1 millones de euros para la gestión de residuos, modernización de líneas ferroviarias, depuración y tratamiento de aguas residuales y preparación para la aplicación de directivas europeas sobre las aguas urbanas residuales y asistencia técnica; en Letonia 219,7 millones de euros para la gestión de residuos, construcción de carreteras, modernización de la red ferroviaria y asistencia técnica; en Lituania 143,6 millones de euros para la modernización de las telecomunicaciones, desarrollo de los sistemas regionales de gestión de residuos y asistencia técnica; en Polonia 1.402 millones de euros para la construcción de plantas depuradoras de aguas residuales, reparación y modernización de líneas ferroviarias, construcción de autopistas y carreteras; en la república eslovaca, 172,5 millones de euros para la modernización y ampliación de una planta regional de depuración de 
aguas residuales, construcción de autopistas, modernización de la red ferroviaria y asistencia técnica; en Eslovenia 45 millones de euros para la construcción de una red de abastecimiento de agua y de plantas regionales de depuración y modernización de una línea ferroviaria. ${ }^{19}$

\section{SAPARD: Programa de Adhesión para el Desarrollo Agrícola y Rural}

La agricultura sigue teniendo mayor peso específico económico en los países candidatos que en los de la Unión Europea, contribuyendo a su PIB y empleando un 22\% de la mano de obra (unos 9,5 millones de personas frente a los 8,2 millones de la Unión Europea de los "quince"). Con la ampliación, la zona de explotación agraria de la UE se ampliará unos 60 millones de hectáreas de muy diversa calidad de terreno y condiciones climáticas. El carácter sensible de la agricultura en las futuras ampliaciones deriva del volumen del sector agrario en algunos PECO y de su considerable potencial de producción agraria.

La complejidad de avanzar en el proceso de ampliación, y la inminente reforma de la PAC que en la actualidad consumen el $46 \%$ del presupuesto comunitario, junto con la actitud renuente de países como Alemania o el Reino Unido a incrementar las aportaciones nacionales a dichos presupuestos hace que en julio de 2002, ninguno de los países candidatos a la ampliación haya cerrado el capítulo 7 de las negociaciones, el referido a la agricultura y que constituye uno de los últimos en resolverse. ${ }^{20}$

No obstante, la importancia socio económica de la agricultura en el Este de Europa y los desequilibrios que ha sufrido como consecuencia de

19 Datos obtenidos de Inforegio News, marzo de 2002. Como se desprende del texto principal entre las inversiones más relevantes y reiteradas en todos los países de la unión se encuentran las infraestructuras relacionadas con la depuración de aguas residuales urbanas. Se trata de facilitar el cumplimiento y la ejecución de la Directiva 91/271/CEE del Consejo de 21 de mayo de 1991 modificada por la Directiva 98/15/CE de 27 de febrero de 1998, que como hemos apuntado con anterioridad constituye una de las principales dificultades en la adaptación del acervo comunitario por parte de los países candidatos.

20 El capítulo 7 fue abierto en las negociaciones tendentes a integrar el acervo comunitario a partir de marzo de 2000, y algún país como Rumania aún no lo ha abierto en junio de 2002. Por lo que respecta a las posiciones de los Estados miembros con anterioridad a la Cumbre de Sevilla, varían desde los que pretenden una integración "normal" de la agricultura, hasta los que, como Reino Unido y Alemania, pretenden que ni siquiera se considere parte del acervo comunitario. La postura intermedia, favorecida por España, de que, pese a la consideración, dentro del acervo no se integre de forma inmediata, no ha logrado superar el bloqueo ni abrir la vía de negociación con vistas a la Cumbre de Bruselas de octubre de 2002. 
los cambios estructurales que se han producido a partir de 1989, han requerido de un apoyo financiero comunitario. Existe un instrumento específico de ayuda comunitaria a la adhesión, con medidas comunitarias a favor de la agricultura y del desarrollo rural en los países candidatos de Europa Central y del Este durante el periodo de preadhesión, para resolver problemas de adaptación a largo plazo del sector agrícola y zonas rurales y para ayudar en la aplicación del acervo en materia de política agrícola común. Se conoce como SAPARD, y su gestión está totalmente descentralizada desde el 2000 en función de los planes de desarrollo rural de los países candidatos, que han sido aprobados por la comisión en el 2000. SAPARD financia proyectos de desarrollo rural seleccionados por cada país, gestionados por las respectivas Agencias SAPARD (acreditadas por las autoridades nacionales competentes y aprobadas por la comisión).

Los informes periódicos de la UE señalan que los países candidatos están realizando muchos avances en la adopción del acervo comunitario en materia agrícola y la convergencia de las políticas nacionales en vigor con la política agrícola común. Sin embargo, queda mucho por hacer respecto de las reformas estructurales como el tamaño de las explotaciones, el sector de la transformación, los circuitos de comercialización y la propiedad de la tierra. La comisión ha señalado en su documento estratégico de 2000 que Polonia y Rumania son los países donde tales reformas estructurales son más necesarias. Además los países candidatos también deben incrementar sus esfuerzos para adoptar el acervo en los sectores veterinario y fitosanitario, así como en la seguridad alimentaria y controles sobre la cadena alimenticia. La ayuda a la preadhesión en materia agraria sirve para ayudar a los países candidatos a transformar y modernizar su sector agrícola y sus regiones rurales para adoptar el acervo de política agraria común.

\section{CONCLUSIONES}

Al aceptar la ampliación a los países del Este, la Unión Europea debe asumir una mayor diferenciación interna y sus implicaciones para el proyecto europeo de convergencia y conversión. La búsqueda de soluciones y nuevas fórmulas será crucial para formular un nuevo proyecto europeo de cohesión y convergencia creíble a la vista de las grandes disparidades 
de riqueza y oportunidades, pero además deberá contar con el apoyo de todos los europeos como un modelo deseable y sostenible de cara al futuro.

Las negociaciones para la adhesión deberán avanzar siguiendo los planes y prioridades señalados por la comisión a medida que son aceptados por los sucesivos consejos europeos que le dan el impulso político. las condiciones clave para que avancen las negociaciones siguen siendo la incorporación del acervo a la legislación de los países candidatos y la adaptación de su capacidad para ejecutarlo y aplicarlo de manera efectiva. Este planteamiento permitirá concluir las negociaciones en el transcurso del año 2002 con los países candidatos que cumplan todos los criterios para la adhesión, permitiendo así a la unión recibir a los nuevos Estados miembros a partir de esa fecha. De este modo, la comisión seguirá supervisando los compromisos de los países negociadores. Las asociaciones para la adhesión continúan siendo los instrumentos centrales de la estrategia de preadhesión, y son la base para programar la asistencia de preadhesión hasta el momento de la incorporación que previsiblemente permitirá la entrada en enero de 2004 de diez nuevos miembros en la unión. La Conferencia Europea deberá seguir siendo el marco para discutir el futuro de la unión con los países candidatos.

Es importante tener muy en cuenta los estudios y las comunicaciones de la Comisión Europea para calmar los temores que pueda producir la ampliación. Sólo una información rigurosa puede servir de base para asentar el consenso y el apoyo activo de los ciudadanos europeos a este reto, que no finaliza con la firma de tratados de adhesión, sino que requerirá de varias décadas para su consolidación.

La Europa ampliada del futuro contará con unos 500 millones de personas con gran variedad étnica, social, jurídica, geográfica y económica. Hace más imprescindible que nunca tener en cuenta los principios básicos de la Unión Europea, como son la proximidad a los ciudadanos, la subsidiariedad y la proporcionalidad. Es preciso procurar que Europa con las futuras adhesiones no ceda a la tentación de reforzar las estructuras centralistas, y sobre el estado de la descentralización en los países candidatos a la adhesión, debe contribuir a una mejor comprensión de las políticas, de las regiones y ciudades de los futuros Estados miembros de la unión. El debate sobre la nueva política de cohesión a partir del 2006 debe ofrecer propuestas claras y detalladas, así como opciones sobre las cuales habrá que reflexionar. Con la ampliación, la mundialización de la economía y el desarrollo de la sociedad de la información, la unión se enfrenta a 
cambios económicos y sociales sin precedentes. Por consiguiente, es necesario que las demás políticas de la comunidad aumenten su contribución a la cohesión económica y social, tal como está previsto en el tratado.

\section{BIBLIOGRAFÍA}

BEST, E., "The Treaty of Nice: Not Beautiful but it'll do", Eipascope European Institute of Public Administration, 2001/1.

Comisión Europea, Actes du Deuxiéme Forum Européen sur la Cohesion, Bruselas, 21-22 de mayo de 2001.

-, Agenda 2000, Luxemburgo, 1997.

-, Las Acciones Estructurales 2000-2006, Luxemburgo, 2000.

, "Participación de los países candidatos en los programas, agencias y comités comunitarios", Comunicación al Consejo, Bruselas 1999.

-, Documento Estratégico sobre la Ampliación, Bruselas, 2001.

, Documento de Síntesis: Informes sobre los Progresos de cada uno de los Países Candidatos en el Camino de la Adhesión, Bruselas, 1999.

, Conseguir que la Ampliación sea un Éxito. Documento de Estrategia e Informe de la Comisión Europea sobre los Progresos de cada uno de los Países Candidatos en la Vía de la Ampliación, Documento Sec (2001) 1744 a 1756; COM (2001) 700 final, Bruselas 13.11.2001.

Comité Económico y Social, Dictamen sobre el Futuro de la PAC, Bruselas, marzo 2002.

Committee of the Regions, Regional and Local Government in the European Union. Responsibilities and Resources (CdR-Studies 1/2001), European, Luxemburgo, 2001.

Consejo Europeo, "Preparar al consejo para la ampliación", Anexos al Consejo Europeo de Gotemburgo (9518/01 + ADD REV 1).

- Conclusiones de la Presidencia, Madrid, 15 y 16 de marzo de 2002.

, Conclusiones de la Presidencia, Laeken, 14 y 15 de diciembre de 2001 (Documento DOC/01/18); Declaración de Laeken, Laeken, 15 de diciembre de 2001 (Documento DOC/01/18- Anexo).

, Conclusiones de la Presidencia, Gotemburgo, 15 y 16 de junio de 2001 (Documento Nr: 200/01). 
, Conclusiones de la Presidencia, Niza, 7, 8 y 9 de diciembre de 2000 (SN 400/00/ES).

, Conclusiones de la Presidencia, Lisboa, 23 y 24 de marzo de 2000 (DOC/00/8).

, Conclusiones de la Presidencia, Helsinki, 10 y 11 de diciembre de 1999 (SN 300/99/ES).

, Conclusiones de la Presidencia, Colonia, 3 y 4 de junio de 1999 (DOC/99/2).

, Conclusiones de la Presidencia, Berlín, 24 y 5 de marzo de 1999 (SN/100/99).

, Conclusiones de la Presidencia, Madrid, 1995 (SN 400/1/REV 1).

-, Conclusiones de la Presidencia, Copenhague, 1993.

DAMMEYER, M., "Introducción”, La Unión Europea ante la perspectiva de la ampliación. La descentralización en los países candidatos para una adhesión inminente, Luxemburgo, 2000.

Dirección General de Política Regional, Comisión Europea, "Ispa cumple su segundo aniversario", Inforegio News, núm. 96, marzo de 2002, p. 1.

—_, "Aprobación del informe intermedio sobre la cohesión", Inforegio News, núm. 95, febrero de 2002.

Dirección General para la Energía (y el Transporte), Comisión Europea, 2000-Annual Review, Bruselas, enero 2001.

_- European Union Energy Outlook to 2020, Energy in Europe, Special Issue, Bruselas, 1999.

GRUBER, K., "Regionalism, nationalism, integration: Central and Western perspectives", Chalenge European on Line Journal, 17.01.2001 (http://www.theepc.be/Challenge_Europe/text/memo.asp)

LOUIS, J. V., "La réforme des institutions de l'Union européenne", Revue du Marché Commun, núm. 4433, diciembre de 2000.

Molina DEL Pozo, C. F., Manual de derecho de la Comunidad Europea, 3a. ed., Madrid, 1997.

PÉREZ CARRILlo, E., "El Tratado de Amsterdam. Entre el mercado de los quince y el siglo XXI", Noticias de la Unión Europea, núm. 189, 2000.

, "El Tratado de Niza entre la consolidación de la UE y el debate sobre el futuro de Europa", Anuario Mexicano de Derecho Internacional, vol. II, 2002.

PRODI, R., “2000-2005. Hacer la nueva Europa”, Boletín de la Unión Europea, Luxemburgo, suplemento 1, 2000. 
RAUX, J., "Après Nice: Pour une approche structurante de la constitutionalisation de l'Union Européenne", en VILÁ CostA, B., "El horizonte institucional de la UE tras la conferencia intergubernamental (de Biarritz a Niza)", Cuaderns de Treball, núm. 38, noviembre de 2001 pp. 7 a 24.

Scientific and Technological Options Assessment, European Parliament, Directorate General for Research, Directorate A, Division for Industry, Research, Energy and Stoa, The consequences of enlargement for EU agriculture, Final Study, Luxemburgo, octubre de 2001.

SCHUMAn, R., Pour L'Europe, París, 1963.

Solana, J., Preparing the Council for Enlargement, Report by Javier Solana, Bruselas, 11 de marzo de 2002.

WeBER, M. et al., "The Wider Picture Enlargement and Cohesion in Europa", The Futures Project, IPTS, Joint Research Centre, 1999. 